\title{
Aetherizing Lambda: Barotropic Fluids as Dark Energy
}

\author{
Eric V. Linder \\ Berkeley Lab \& University of California, Berkeley, CA 94720 \\ Robert J. Scherrer \\ Department of Physics and Astronomy, Vanderbilt University, Nashville, TN 37235
}

(Dated: October 24, 2018)

\begin{abstract}
We examine the class of barotropic fluid models of dark energy, in which the pressure is an explicit function of the density, $p=f(\rho)$. Through general physical considerations we constrain the asymptotic past and future behaviors and show that this class is equivalent to the sum of a cosmological constant and a decelerating perfect fluid, or "aether", with $w_{A E} \geq 0$. Barotropic models give substantially disjoint predictions from quintessence, except in the limit of $\Lambda$ CDM. They are also interesting in that they simultaneously can ameliorate the coincidence problem and yet "predict" a value of $w \approx-1$.
\end{abstract}

\section{INTRODUCTION}

Observational evidence strongly points to an accelerated expansion of the universe [1, 2], but the physical origin of this acceleration is unknown. Since general relativity relates spacetime curvature (and hence acceleration) to energy, it is natural to hypothesize that either this relationship must be modified (as in extended gravity models [3, 4] ), or that there is some additional source of energy density driving the expansion. Many models in the latter category have been proposed, including scalar field models ("quintessence") [5, 6, 7, 8, 9, 10], scalar field models with modified kinetic terms ("k-essence") [11, 12, 13, 14, 15, 16, 17, 18, 19, 20], and even more exotic possibilities (see, for example, the review of [21]).

Here we examine in more detail a class of models called barotropic fluids, in which the dark energy pressure $p_{D E}$ is given as an explicit function of the density $\rho_{D E}$. This can be viewed as a very simple prescription, in contrast to other models (such as the ones above) where the relation is implicit, written in terms of intermediate variables. Although some barotropic models are well studied, we find here a number of new, general properties that makes this class of interest in dark energy physics. Specific models investigated previously include the Chaplygin gas [22, 23] and the generalized Chaplygin gas [22, 24], the linear equation of state [25, 26, 27, 28] and the affine equation of state 29, 30] (note these are actually the same model), the quadratic equation of state [29, 31], and the van der Waals equation of state [32, 33]. Such models have been considered either as unified models for dark matter and dark energy together, or as models for the dark energy alone. We confine our attention to the latter case.

One of the key properties of barotropic fluids is that the sound speed, $c_{s}^{2}=d p_{D E} / d \rho_{D E}$, does not have to equal the speed of light as in quintessence models. In addition, the condition $c_{s}^{2} \geq 0$ causes the barotropic dynamical behavior to be distinguishable from quintessence - that is, they tend to lie in distinct regions of the equation of state phase space [34]. Here we extend these results to a more general discussion of the types of behav- ior that are allowed for barotropic models. We will see that limits $0 \leq c_{s}^{2} \leq 1$ on the sound speed, along with some fairly general observational constraints, allow surprisingly broad conclusions to be made about the properties of viable barotropic models, ruling out some models in the literature. Moreover, the properties describe an attractively simple picture of dark energy, together with a possible simultaneous resolution of the coincidence problem and why today the equation of state $w$ is near -1 .

We discuss general properties of barotropic fluids in \II, together with some special cases. In $₫$ III we explore the distinction between barotropic and scalar field solutions to the dark energy puzzle, and the relation to $\Lambda \mathrm{CDM}$, finishing with a comparison of the coincidence and $w \approx-1$ behaviors of the different classes.

\section{PROPERTIES OF A BAROTROPIC FLUID}

\section{A. General Properties}

We define a barotropic fluid as any fluid in which the physics of the fluid is fully determined by the pressure as an explicit function of the density:

$$
p_{D E}=f\left(\rho_{D E}\right) .
$$

Thus, the equation of state function $f$ completely characterizes a barotropic fluid. For example, the generalized Chaplygin gas has the equation of state function [24]

$$
p_{D E}=-\frac{A}{\rho_{D E}^{\alpha}},
$$

where $A$ and $\alpha$ are constants, while the quadratic equation of state is

$$
p_{D E}=p_{0}+\alpha \rho_{D E}+\beta \rho_{D E}^{2},
$$

with the linear (or affine) model corresponding to the special case $\beta=0$. The van der Waals equation of state 
is 32,33 .

$$
p_{D E}=\frac{\gamma \rho_{D E}}{1-\beta \rho_{D E}}-\alpha \rho_{D E}^{2}
$$

How do barotropic models differ from quintessence models for dark energy? For a canonical, minimally coupled scalar field $\phi$, the relation between pressure and density is given parametrically by

$$
\begin{aligned}
& p_{\phi}=\frac{1}{2} \dot{\phi}^{2}-V(\phi), \\
& \rho_{\phi}=\frac{1}{2} \dot{\phi}^{2}+V(\phi),
\end{aligned}
$$

where $\dot{\phi}=d \phi / d t$, and $V(\phi)$ is the quintessence potential.

One can certainly find quintessence potentials for which $p_{\phi}$ is not a single-valued function of $\rho_{\phi}$ and which therefore can never be described in terms of equation (1). For instance, a field oscillating about the minimum of a potential has zero kinetic term at either extreme of the oscillation and zero potential term at the minimum (in terms of the equation of state ratio $w=p_{\phi} / \rho_{\phi}$, this is $w=-1$ and +1 respectively). Thus, $p_{\phi}$ passes through 0 twice on every oscillation, with a decreasing value of $\rho_{\phi}$ each time (since $\rho_{\phi}$ diminishes as the universe expands). On the other hand, many quintessence models can be characterized by a pressure which is a single-valued function of density, so what distinguishes these models from the barotropic models we consider here ${ }^{1}$

The main distinction is that barotropic models have a value of $d p_{D E} / d \rho_{D E}$ which is constrained by limits on the sound speed. The sound speed for a barotropic fluid is given by

$$
c_{s}^{2}=\frac{d p_{D E}}{d \rho_{D E}} .
$$

Note that there is no need to write partial derivatives, since $p_{D E}$ depends only on $\rho_{D E}$. To ensure stability, we must have $c_{s}^{2} \geq 0$, so that $d p_{D E} / d \rho_{D E} \geq 0$. Thus, the function $f\left(\rho_{D E}\right)$ in equation (11) is not arbitrary; it must satisfy $d f / d \rho_{D E} \geq 0$. We will further require, for causality, that $c_{s}^{2} \leq 1([35]$, but also see [36] $)$. This imposes the additional constraint $d f / d \rho_{D E} \leq 1$.

In contrast, a canonical minimally coupled scalar field is an imperfect fluid. While its adiabatic sound speed is given by equation (7) (written as a partial derivative holding the entropy, or scale factor, fixed), its physical sound speed is always equal to the speed of light.

${ }^{1}$ Indeed, it is trivial to write down an effective potential for a barotropic model:

$$
\begin{aligned}
V(\phi) & =(\rho-f) / 2 \\
\phi & =\int d t(\rho+f)^{1 / 2},
\end{aligned}
$$

and from this $V(\phi)$ alone one could not tell if the physics was barotropic or quintessential.
Thus, these models are not subject to the constraint that $d p / d \rho \geq 0$; in fact, they generically have $d p / d \rho<0$ 34, 37]. This is the reason that barotropic models and many quintessence models occupy disjoint regions in the $w-w^{\prime}$ phase plane [34]. (For more general discussions of the behavior of scalar field dark energy models in the $w-w^{\prime}$ phase space, see [37, 38, 39, 40]).

Starting from the definition of the equation of state ratio, $w=p_{D E} / \rho_{D E}$, and taking the derivative with respect to the logarithmic scale factor $\ln a$, denoted by a prime, one has

$$
\begin{aligned}
w^{\prime} & =-3(1+w)\left(\frac{d p_{D E}}{d \rho_{D E}}-w\right) \\
& =-3(1+w)\left(c_{s}^{2}-w\right) .
\end{aligned}
$$

The requirement that $c_{s}^{2} \geq 0$ then gives 34 .

$$
w^{\prime} \leq 3 w(1+w) .
$$

Here we consider only barotropic models for which $w>$ -1 , although it is also possible to generate barotropic phantom models with $w<-1$ [41, 42, 43]. Since, for dark energy, $w<0$, we have $w^{\prime}<0$ for all barotropic fluids that can serve as dark energy. Models of this kind, in which $w$ approaches -1 with time, have been dubbed "freezing" models [37], although for quintessence freezing models one frequently has the opposite of equation (10): $w^{\prime} \geq 3 w(1+w)$. (Quintessence models can be found to violate this, while barotropic models will never break Eq. 10). Further, the upper bound on the sound speed, $c_{s}^{2} \leq 1$, gives a lower bound on $w^{\prime}$ :

$$
w^{\prime} \geq-3(1+w)(1-w) .
$$

This is precisely the null line for the $w-w^{\prime}$ phase plane 38] and leads to the one exception where quintessence models are exactly equivalent to barotropic models: skating models [38, 44], with kinetic energy along a flat potential, follow the equality in equation (11) and correspond to barotropic models with $f\left(\rho_{D E}\right)=\rho_{D E}-\rho_{\star}$; both have $c_{s}^{2}=1$. This is a pathological case, however, as the kinetic energy of skating models redshifts as $a^{-6}$ and the model quickly becomes indistinguishable from a cosmological constant.

Since $w$ decreases with time, equation (8) has a generic future attractor at $w=-1$, independent of the functional form of $f\left(\rho_{D E}\right)$. At the attractor, the density, pressure, and sound speed asymptotically approach constant values, which we denote $\rho_{\infty}, p_{\infty}$, and $c_{s \infty}$. Taking $c_{s}=c_{s \infty}$ in equation (9), we see that $w$ approaches -1 as

$$
1+w \sim a^{-3\left(1+c_{s \infty}^{2}\right)}
$$

and the dark energy density asymptotically approaches $\rho_{\infty}$ as

$$
\rho_{D E}-\rho_{\infty} \sim a^{-3\left(1+c_{s \infty}^{2}\right)} .
$$


Since at late times the density always approaches a constant, $\rho_{\infty}$, this suggests decomposing the barotropic energy density into two components,

$$
\rho_{D E}=\rho_{\infty}+\rho_{A E},
$$

where the first term represents an always present cosmological constant and the second term defines an "aether" density, $\rho_{A E}$, a spatially pervasive fluid with sound speed generally different from unity.

The aether component is itself barotropic (with zero cosmological constant piece), and by comparing equations (14) and (13) to the usual behavior of some component $x, \rho_{x} \sim a^{-3\left(1+w_{x}\right)}$, one sees that $0 \leq w_{A E} \leq 1$. That is, we can represent any barotropic fluid overall as consisting of a cosmological constant plus a positive equation of state perfect fluid. The sound speed of the aether is equal to the sound speed of the full barotropic component, a result that is easily proven using the formulas for summing components, equations (26) and (27) of [40], together with equation (9).

Note that the cosmic acceleration from the dark energy arises purely from the cosmological constant piece. In contrast to the barotropic dark energy as a whole, the aether component acts to decelerate and has a density that decreases at least as fast as the matter density, $\rho_{M} \sim$ $a^{-3}$, and no more rapidly than a stiff fluid, $\rho \sim a^{-6}$.

As a specific example, consider the Chaplygin gas model, which is given by equation (2) with $\alpha=1$. The full Chaplygin gas density evolves as

$$
\rho_{D E}=\sqrt{\rho_{\infty}^{2}+\left(\rho_{D E, 0}^{2}-\rho_{\infty}^{2}\right) a^{-6}},
$$

with the future attractor at $\rho_{\infty}=\sqrt{A}$, so the aether density is given by

$$
\rho_{A E}=\sqrt{\rho_{\infty}^{2}+\left(\rho_{D E, 0}^{2}-\rho_{\infty}^{2}\right) a^{-6}}-\rho_{\infty} .
$$

The aether density decays as $a^{-3}$ at early times (i.e. $\left.w_{A E}=0\right)$, while in the limit where the constant-density attractor is approached, $\rho_{A E}$ decays as $a^{-6}$ (i.e. $w_{A E}=$ $+1)$.

Requiring a matter dominated era at high redshift constrains the behavior of the aether component at early times. Since $w_{A E} \geq 0$, the energy density of this component will tend to dominate in the past. However such an aether component with a density greater than the matter density at high redshift violates a host of observational constraints [45]. Thus, we must have $w_{A E} \rightarrow 0$ as $a \rightarrow 0$. Furthermore, applying equation (9) to the aether component, we see that as $a \rightarrow 0$ we have $c_{s}^{2} \leq w_{A E}$ so in this limit $c_{s}^{2} \rightarrow 0$.

These arguments then allow us to put very general constraints on the behavior of the overall barotropic dark energy: it will always behave like a pressureless dust component at early times, and like a cosmological constant at late times. (Note that "late times" could mean the far future, rather than the present). In order to produce dust-like behavior at early times, the functional form of $f\left(\rho_{D E}\right)$ must satisfy the constraint $d f / d \rho_{D E} \rightarrow 0$ as $\rho_{D E} \rightarrow \infty$, as well as the previously-discussed soundspeed limits: $0 \leq d f / d \rho_{D E} \leq 1$ at all times. We discuss the observational implications of this further in $\amalg$

For the barotropic models we have mentioned, these limits impose severe constraints. For example, for the quadratic and affine models of equation (3), our limits impose $\beta=0$ and $\alpha=0$, so all acceptable dark energy models of this type reduce to the simple case $p_{D E}=$ constant. This corresponds to the special case where $\rho_{A E}$ behaves exactly like dust at all times (see the next section). Similarly, the van der Waals model (equation 44) is found to have unphysical behavior. For the generalized Chaplygin gas (equation 2), our constraints give $\alpha>0$, ruling out several extensions of the generalized Chaplygin gas [46].

The aether decomposition also provides a simple recipe for producing acceptable barotropic models. Specifically, every barotropic equation of state can be written in the form

$$
p_{D E}=-\rho_{\infty}+g\left(\rho_{D E}-\rho_{\infty}\right),
$$

where the function $g$ is subject to the constraints

$$
\begin{gathered}
0 \leq d g(\rho) / d \rho \leq 1, \\
g(0)=0,
\end{gathered}
$$

and, in the limit where $\rho \rightarrow \infty$,

$$
d g(\rho) / d \rho \rightarrow 0 .
$$

\section{B. Special Cases}

Now consider some special cases of interest. When $c_{s}^{2}=0$, we have the previously-mentioned constant pressure model, characterized by $p=-\rho_{\infty}$. The density in this case evolves as

$$
\rho=\rho_{\infty}+C a^{-3},
$$

i.e., this looks just like the $\Lambda \mathrm{CDM}$ model (though with an additional matter contribution). This model has been previously discussed elsewhere. For instance, it corresponds to the $\alpha=0$ limit of the generalized Chaplygin gas [24], and it is a special case of the "mocker models" discussed in [38], in which it was noted that such models are characterized by $w^{\prime}=3 w(1+w)$, defined there as the constant pressure line.

For $c_{s}^{2}$ constant but nonzero, we have

$$
\rho=\rho_{\infty}+C a^{-3\left(1+c_{s}^{2}\right)} .
$$

These models are all observationally excluded as noted earlier, since they asymptotically dominate the expansion at high redshift. In particular, pure skating models have $c_{s}^{2}=1$ and therefore correspond to $d g / d \rho=1$ at all times; such models then violate the condition in equation (20) above. 


\section{Relation to k-essence}

Note that there is a one-to-one correspondence between barotropic fluids and the subset of k-essence models with constant potential, the so-called "purely kinetic" k-essence models. The latter models were first investigated in the context of inflation [11] and later proposed as unified models of dark matter and dark energy [19]. These models are characterized by a Lagrangian of the form:

$$
p=F(X)
$$

where $X$ is

$$
X=\frac{1}{2} \nabla_{\mu} \phi \nabla^{\mu} \phi
$$

and $\phi$ is the $\mathrm{k}$-essence scalar field. The pressure in these models is simply given by equation (23), while the energy density is

$$
\rho=2 X(d F / d X)-F .
$$

To convert between a kinetic k-essence model and a barotropic model, one inverts the function $F$ to find $X=F^{-1}(p)$ and substitutes this into equation (25) to convert $\rho(X)$ into $\rho(p)$. Inverting this delivers the barotropic equation (11). If one starts with a barotropic model, one inverts $f$ to give $\rho=f^{-1}(p)$ and interprets equation (25) as a differential equation to solve for $F(X)$. To wit (cf. [47])

$$
\int \frac{d F}{f^{-1}(F)+F}=\ln \left(C X^{1 / 2}\right),
$$

where $C$ is a constant. This one-to-one correspondence (assuming the functions are invertible) means that the trajectories for purely kinetic k-essence lie in the same region of the $w-w^{\prime}$ phase plane as the trajectories for barotropic fluids [20].

For the constant $c_{s}^{2}$ barotropic model, for example, the explicit barotropic relation between pressure and density is

$$
p_{D E}=c_{s}^{2} \rho_{D E}+\left(1+c_{s}^{2}\right) \rho_{\star},
$$

and the analogous k-essence Lagrangian is [20, 30]

$$
p=\rho_{\star}+A \frac{2 c_{s}^{2}}{1+c_{s}^{2}} X^{\left(1+c_{s}^{2}\right) /\left(2 c_{s}^{2}\right)},
$$

where $A$ is an arbitrary constant allowed by field redefinition in $X$.

\section{OBSERVATIONAL SIGNATURES}

Despite the generality of the models discussed here, they do provide some distinctive observational signatures. We first consider features in the homogeneous background properties, e.g. expansion history and equation of state, and then in the spatial perturbation properties.

Consider first the jerk parameter of the expansion history 48.

$$
j \equiv \frac{a^{2} \dddot{a}}{\dot{a}^{3}} .
$$

The importance of this parameter was first emphasized by [49], and it was presented as one of the "statefinder" parameters in [50, 51]. In a flat universe, $j$ is given by 49.

$$
j=1+\frac{9}{2} \Omega_{D E} \frac{d p_{D E}}{d \rho_{D E}}\left(1+w_{D E}\right) .
$$

Thus, for $\Lambda \mathrm{CDM}$ (including the constant pressure models discussed in $\underline{\mathrm{IIB}}_{\text {}}$, we see that $j=1$, independent of the value of $\Omega_{\Lambda}$ [52]. For the barotropic models considered here, the requirement that $d p_{D E} / d \rho_{D E} \geq 0$ translates into a simple bound: $j \geq 1$. On the other hand, for quintessence models, we can write 34,38 .

$$
j=1-\frac{3}{2} \Omega_{D E}\left[w^{\prime}-3 w(1+w)\right]
$$

Since quintessence models generally satisfy $w^{\prime} \geq 3 w(1+$ w) [37], we see that they are often characterized by $j \leq 1$. Thus, accurate measurement of jerk parameter $j<1$ (or equivalently $w$ and $w^{\prime}$ ) could provide perhaps the cleanest observational signature to distinguish barotropic dark energy from quintessence. One can view this as determining the number of internal degrees of freedom in the dark energy physics [30, 53].

Unfortunately, the jerk parameter is rather difficult to derive from current observational data. Assuming a constant value for $j, 54$ derived $j=2.16_{-0.75}^{+0.81}$. While this might naïvely seem to favor barotropic models, in fact the assumption of constant $j$ strongly biases the result. Typical barotropic and quintessence models generally have values of $j$ that vary significantly with time, and current observations are insufficient to distinguish these two types of models.

Note that as $j$ approaches unity, it becomes difficult to distinguish barotropic (or quintessence) models from $\Lambda \mathrm{CDM}$ using the expansion history, since the constantpressure model is degenerate with $\Lambda$ CDM. Two possible observational signatures exist. In the case of barotropic dark energy, some of the contribution to the zero-pressure dark component can arise not only from dark matter but also from the aether component. If the dark matter particle is detected, and its relic abundance can be calculated from its physical properties, then one signature of barotropic dark energy would be an anomalously high observed value of $\Omega_{M}$ in relation to the dark matter (and baryon) calculation.

A second possible signature is the behavior of spatial perturbations. Since $c_{s}^{2}=0$ for the constant-pressure 
barotropic model, one might hope to distinguish it observationally from the corresponding quintessence model with $c_{s}^{2}=1$ on the basis of perturbation growth. (Constraints on the dark energy sound speed have been explored for constant sound speed in [55, 56] ). Interestingly, where barotropic and quintessence models are closest in their dynamics (i.e. near $w^{\prime} \approx 3 w(1+w)$ ), they differ most in sound speed, and where they are closest in sound speed, they differ most in their dynamics. However, as $w \rightarrow-1$, barotropic models and $\Lambda$ CDM become degenerate, at least to linear order. We can see this by examining the equation for linear perturbation growth in the dark energy fluid in synchronous gauge [57, 58]:

$$
\dot{\delta}_{D E}+(1+w)\left(\theta_{D E}+\dot{h} / 2\right)+3 H\left(c_{s}^{2}-w\right) \delta_{D E}=0,
$$

where $h$ is the trace of the perturbation to the FriedmanRobertson-Walker metric, and $\theta_{D E}$ is the divergence of the fluid velocity. The key point is that for the case $w=-1$, there is no growing mode in $\delta_{D E}$, since the last term is always positive. Thus, $w=-1$ models cannot be distinguished, regardless of the value of $c_{s}^{2}$ (this point is emphasized in [58] for the case of the $\alpha=0$ Chaplygin gas and $\Lambda \mathrm{CDM}$ ).

One can also argue qualitatively from equation (32) that the growth of density perturbations should become relatively insensitive to $c_{s}^{2}$ in the limit where $w$ is close to -1 . This conclusion is borne out by detailed comparisons in the $w-c_{s}^{2}$ plane between models and the observations [55, 56]. These investigations show likelihood curves that are nearly independent of $c_{s}^{2}$ for $w$ near -1 .

Note that barotropic models as a class can never be "ruled out" as long as $\Lambda \mathrm{CDM}$ remains viable, since as a limiting case constant-pressure barotropic models include $\Lambda \mathrm{CDM}$. We have shown ways, however, in which one can generally constrain the allowed parameter space for barotropic models. That said, all observationally-allowed barotropic models must approach the constant-pressure model at high redshift, making them indistinguishable from $\Lambda \mathrm{CDM}$ in this limit (save through the theoretical predictions for $\Omega_{M}$ noted above).

One can take this further and argue that barotropic models "predict" a value of $w$ near -1 , in a way that quintessence models do not. Our argument is based on the upper bound on $w^{\prime}$ given by equation (10). This equation shows that, for barotropic models with nonnegative $c_{s}^{2}$, the value of $w$ for the dark energy can never "loiter" at a value between $w=0$ and $w=-1$. While $w$ can lie near 0 for arbitrarily long times in these models, once it begins to decrease toward -1 , equation (10) puts a lower bound on the rate of decrease. Thus, one cannot have arbitrarily long periods in which $w$ has some value between 0 and -1 . The opposite is true in quintessence models; it is easy to construct such models (trackers) with $w$ roughly constant and equal to nearly any desired value [10].

In terms of our aether decomposition, the slowest rate of decrease for $w_{D E}$ occurs when $w_{A E}=0$. In this case, for example, $w$ decreases from -0.1 to -0.9 as the scale factor increases by about a factor of 4 (i.e. within 1.5 e-folds). Other choices for the equation of state function can only produce a more rapid decrease in $w$. Thus, in barotropic models, a value of $w$ between 0 and -1 must always be a transient phenomenon, leading to the argument that barotropic models "predict" a value of $w$ near -1 (a prediction which would have been considerably more convincing had we made it a decade ago). Note that a $w \rightarrow-1$ attractor is also present in some unified models for dark matter and dark energy [59, 60], although such unified models are outside the scope of our discussion.

Since the only viable barotropic models are those that scale like the dominant component at high redshift, no other special selection needs to be applied: in this sense the "bug" of not being able to distinguish a constantpressure barotropic model $\left(w_{A E}=0\right)$ from $\Lambda \mathrm{CDM}$ is really a "feature" of ameliorating the problem of fine tuning initial conditions. (The usual cosmological constant problem remains of why the non-aether part, $\rho_{\infty}$, is so small.)

Note that the coincidence problem is also somewhat ameliorated (see [61] for a recent discussion of the coincidence problem from a novel perspective). For the case of a cosmological constant, the ratio of dark energy density to matter density has to increase by nine orders of magnitude between recombination and today, leading to the question of why dark energy overtakes matter basically now. In contrast, the barotropic models can easily have a more natural-seeming ratio of order $10^{-2}$ or $\mathcal{O}(1)$ at recombination. While quintessential tracking models can also have such ratios, they have difficulties in then achieving $w \approx-1$ today. Thus barotropic models have attractive characteristics with regards to both the fine tuning and coincidence problems. In effect, the aether component of the barotropic fluid anaesthetizes the cosmological constant against the pain of fine tuning.

\section{CONCLUSIONS}

Barotropic fluids have a number of characteristics that make them an interesting class of dark energy models. They have, by definition, an explicit rather than implicit equation of state relating the pressure and the energy density. While this relation is nominally quite general, we show that simple physical conditions such as stability and causality severely restrict the allowed functional forms. In particular, we demonstrate that viable barotropic models must possess the following properties:

- Asymptotic future de Sitter state, where the dynamics freezes to a cosmological constant state,

- Dynamics distinct from much of quintessence, lying in a separate region of $w-w^{\prime}$ phase space; sound speed generally distinct from quintesssence,

- Acts as a sum of a cosmological constant and a perfect fluid "aether" component with $w_{A E} \geq 0$, 
- The aether component must have $w_{A E} \rightarrow 0, c_{s}^{2} \rightarrow 0$ in the past in order not to violate matter domination.

These results both unify a number of special cases in the literature and rule out several models.

We consider several observational signatures to distinguish barotropic fluids through both the effects on background expansion and on perturbation growth. For example, in the barotropic case the sound speed is not restricted to be the speed of light, as in canonical, minimally coupled scalar field models. Constant-pressure $\left(c_{s}^{2}=0\right)$ barotropic models are however degenerate with $\Lambda \mathrm{CDM}$, though possibly distinguishable through a discrepancy between particle physics predictions for the dark matter density and cosmological observations.

Finally, the aether component of the barotropic fluid can anaesthetize the cosmological constant against some of its fine tuning and coincidence problems. In the high redshift universe the dark energy appears like $\Lambda$ CDM, but with a dark component energy density that can be comparable to the matter density. At late times, it naturally and rapidly transitions from a matter-like behavior to behavior that approaches a pure cosmological constant.

\section{Acknowledgments}

E.V.L. was supported in part by the Director, Office of Science, Office of High Energy Physics, of the U.S. Department of Energy under Contract No. DE-AC0205CH11231. R.J.S. was supported in part by the Department of Energy (DE-FG05-85ER40226).
[1] E. Komatsu et al., ApJS 180, 330 (2009).

[2] M. Kowalski et al., ApJ 686, 749 (2008).

[3] G. Dvali, G. Gabdadze, and M. Porrati, Phys. Lett. B 485, 208 (2000).

[4] T.P. Sotiriou and V. Faraoni, arXiv:0805.1726

[5] C. Wetterich, Nucl. Phys. B 302, 668 (1988).

[6] B. Ratra and P.J.E. Peebles, Phys. Rev. D 37, 3406 (1988).

[7] M.S. Turner and M. White, Phys. Rev. D 56, R4439 (1997).

[8] R.R. Caldwell, R. Davé, and P.J. Steinhardt, Phys. Rev. Lett. 80, 1582 (1998).

[9] A.R. Liddle and R.J. Scherrer, Phys. Rev. D 59, 023509 (1999)

[10] P.J. Steinhardt, L. Wang, and I. Zlatev, Phys. Rev. D 59, 123504 (1999).

[11] C. Armendariz-Picon, T. Damour, and V. Mukhanov, Phys. Lett. B 458, 209 (1999).

[12] J. Garriga and V.F. Mukhanov, Phys. Lett. B 458, 219 (1999).

[13] T. Chiba, T. Okabe, M. Yamaguchi, Phys. Rev. D 62, 023511 (2000).

[14] C. Armendariz-Picon, V. Mukhanov, and P.J. Steinhardt, Phys. Rev. Lett. 85, 4438 (2000).

[15] C. Armendariz-Picon, V. Mukhanov, and P.J. Steinhardt, Phys. Rev. D 63, 103510 (2001).

[16] T. Chiba, Phys. Rev. D 66, 063514 (2002).

[17] L.P. Chimento and A. Feinstein, Mod. Phys. Lett. A 19, 761 (2004).

[18] L.P. Chimento, Phys. Rev. D 69, 123517 (2004).

[19] R.J. Scherrer, Phys. Rev. Lett. 93, 011301 (2004).

[20] R. de Putter and E.V. Linder, Astropart. Phys. 28, 263 (2007)

[21] E.J. Copeland, M. Sami, and S. Tsujikawa, Int. J. Mod. Phys. D 15, 1753 (2006).

[22] A.Y. Kamenshchik, U. Moschella, and V. Pasquier, Phys. Lett. B 511, 265 (2001).

[23] N. Bilic, G.B. Tupper, and R.D. Viollier, Phys. Lett. B 535, 17 (2002).

[24] M.C. Bento, O. Bertolami, and A.A. Sen, Phys. Rev. D
66, 043507 (2002).

[25] T. Chiba, N. Sugiyama, and T. Nakamura, MNRAS 289, L5 (1997).

[26] T. Chiba, N. Sugiyama, and T. Nakamura, MNRAS 301, 72 (1998)

[27] E. Babichev, V. Dokuchaev, and Yu. Eroshenko, Class. Quant. Grav. 22, 143 (2005).

[28] R. Holman and S. Naidu, astro-ph/0408102

[29] K.N. Ananda and M. Bruni, Phys. Rev. D 74, 023523 (2006).

[30] C. Quercellini, M. Bruni, and A. Balbi, Class. Quant. Grav., 24, 5413 (2007).

[31] S. Nojiri and S.D. Odintsov, Phys. Rev. D 70, 103522 (2004).

[32] G.M. Kremer, Phys. Rev. D 68, 123507 (2003).

[33] S. Capozziello et al., JCAP 0504, 005 (2005).

[34] R.J. Scherrer, Phys. Rev. D 73, 043502 (2006).

[35] G.F.R. Ellis, R. Maartens, and M.A.H. MacCallum, Gen. Rel. Grav. 39, 1651 (2007).

[36] E. Babichev, V. Mukhanov, and A. Vikman, JHEP 02, 101 (2008).

[37] R.R. Caldwell and E.V. Linder, Phys. Rev. Lett. 95, 141301 (2005).

[38] E.V. Linder, Phys. Rev. D 73, 063010 (2006).

[39] T. Chiba, Phys. Rev. D 73, 063501 (2006).

[40] E.V. Linder, Gen. Rel. Grav. 40, 329 (2008)

[41] H. Stefancic, Phys. Rev. D 71, 084024 (2005).

[42] S. Nojiri, S.D. Odintsov, and S. Tsujikawa, Phys. Rev. D 71, 063004 (2005).

[43] S. Capozziello, V.F. Cardone, E. Elizalde, S. Nojiri, S.D. Odintsov, Phys. Rev. D 73, 043512 (2006).

[44] M. Sahlén, A.R. Liddle, and D. Parkinson, Phys. Rev. D 72, 083511 (2005)

[45] M. Doran, G. Robbers, and C. Wetterich, Phys. Rev. D 75, 023003 (2007)

[46] A.A. Sen and R.J. Scherrer, Phys. Rev. D 72, 063511 (2005).

[47] A. Díez-Tejedor and A. Feinstein, Int. J. Mod. Phys. D 14, 1561 (2005).

[48] M. Visser, Class. Quant. Grav. 21, 2603 (2004). 
[49] T. Chiba and T. Nakamura, Prog. Theor. Phys. 100, 1077 (1998).

[50] V. Sahni, T.D. Saini, A.A. Starobinsky, and U. Alam, JETP Lett. 77, 201 (2003).

[51] U. Alam, V. Sahni, T.D. Saini, and A.A. Starobinsky, MNRAS 344, 1057 (2003).

[52] R.D. Blandford, M. Amin, E.A. Baltz, K. Mandel, and P.J. Marshall, astro-ph/0408279

[53] R.R. Caldwell, private communication.

[54] D. Rapetti, S.W. Allen, M.A. Amin, and R.D. Blandford, MNRAS 375, 1510 (2007).

[55] R. Bean and O. Doré, Phys. Rev. D 69, 083503 (2004).
[56] S. Hannestad, Phys. Rev. D 71, 103519 (2005).

[57] S. Veeraraghavan and A. Stebbins, Astrophys. J. 365, 37 (1990).

[58] P.P. Avelino, L.M.G. Beca, J.P.M. de Carvalho, and C.J.A.P. Martins, JCAP 0309, 002 (2003).

[59] M.C. Bento, O. Bertolami, and A.A. Sen, Phys. Rev. D 70, 083519 (2004).

[60] D. Bertacca, S. Matarrese, and M. Pietroni, Mod. Phys. Lett. A 22, 2893 (2007).

[61] C.A. Egan and C.H. Lineweaver, Phys. Rev. D 78, 083528 (2008). 